\title{
Theory of Nanocluster Size Distributions from Ion Beam Synthesis
}

\author{
C. W. Yuan, ${ }^{1,2}$ D. O. Yi, ${ }^{1,2}$ I. D. Sharp ${ }^{3}$ S. J. Shin, ${ }^{1,2}$ C. Y. Liao,,${ }^{1,2}$ \\ J. Guzman, ${ }^{1,2}$ J. W. Ager, III, ${ }^{2}$ E. E. Haller, ${ }^{1,2}$ and D. C. Chrzan ${ }^{1,2}$ \\ ${ }^{1}$ Department of Materials Science and Engineering, \\ University of California, Berkeley, CA, 94720 \\ ${ }^{2}$ Materials Science Division, Lawrence Berkeley \\ National Laboratory, Berkeley, CA 94720-1760 \\ ${ }^{3}$ Walter Schottky Institut, Technische Universität München, \\ Am Coulombwall 3, 85748 Garching, Germany
}

(Dated: April 22, 2009)

\begin{abstract}
Ion beam synthesis of nanoclusters is studied via both kinetic Monte Carlo simulations and the self-consistent mean-field solution to a set of coupled rate equations. Both approaches predict the existence of a steady state shape for the cluster size distribution that depends only on a characteristic length determined by the ratio of the effective diffusion coefficient to the ion flux. The average cluster size in the steady state regime is determined by the implanted species/matrix interface energy.

PACS numbers: 81.07.-b,81.15.Jj,61.80.-x,61.72.U-
\end{abstract}


The study of nucleation, growth and coarsening has been the focus of research for many years [1]. For 2-D nucleation and growth, it has been established that scaling solutions describe the evolution of the cluster size distributions during deposition [2-6]. The properties of these solutions can be used to determine the diffusion coeffiecient for adatoms, $D[7,8]$. Further, the shape of the 2-D cluster size distribution is governed by the critical cluster size governing nucleation[5]: Larger critical cluster sizes lead to narrower cluster size distributions. Thus the basic physics governing submonolayer epitaxy is well established with the minimal set of important parameters identified and measured experimentally.

A 3-D analogue of epitaxial growth can be found in the ion beam synthesis (IBS) of nanocrystals. IBS begins with the implantation of insoluble ions into a matrix. As the ions are implanted, they move about and encounter other implanted ions. These collisions lead to cluster nucleation and growth.

IBS differs from 2-D growth in an important respect: During IBS, cluster growth is often interrupted by ion damage. The deposition of energetic ions fragments the clusters, leads to inverse Ostwald ripening [9], and ultimately limits cluster sizes. The basic understanding of IBS has been exploited to fabricate interesting nanostructures [9-15] and alter cluster size distributions $[15,16]$. But unlike low dose semiconductor doping via implantation that is extensively studied and understood [17-20], no detailed, comprehensive, quantitative theory if IBS has been developed. Further, in contrast to the 2-D problem, the minimal set of parameters required to predict the shape of the cluster-size distributions arising from IBS have not been determined.

In this paper, kinetic Monte Carlo (KMC) simulations, and the mean-field self-consistent solution to a set of coupled rate equations are developed to model cluster size distributions arising from IBS. Because growth and coarsening under annealing is well understood $[1,21]$, the present study focueses solely on the size distribution evolution during implantation, which seeds the annealing stage. It is shown that the two key parameters governing the as-implanted size distribution for a given material are the characteristic length, $L \equiv\left(D n_{\infty} / F\right)^{1 / 2}$, where $F$ is the volumetric flux and $\mathrm{n}_{\infty}$ is the ion's solubility, and the interface energy between the implanted species and the matrix.

The employed KMC simulations include five fundamental processes: 1) implantation into an amorphous silica matrix at a rate $F, 2$ ) the off-lattice random walk of implanted atoms, represented by a diffusion coefficient $D$ (the hop distance is chosen to be $5 \AA$ ), 3 ) the 
attachment of atoms to each other and existing clusters followed by immediate relaxation of all clusters to a spherical shape, 4) the thermally driven detachment of atoms from existing clusters, and 5) the ion damage induced fragmentation of clusters.

The binding of atoms to clusters (and each other) is modeled as described in reference [21]. The radius of an atom is chosen to be $r_{a}=(3 \Omega / 4 \pi)^{1 / 3}$, with $\Omega$ the equilibrium atomic volume of the implanted species. An atom binds with another implanted atom or existing cluster when the two "touch." In order to accelerate the simulations, the atomic scale structure of the clusters is not stored. Instead, all clusters are assumed to relax immediately to a spherical shape with the volume equal to the number of atoms in the cluster multiplied by the atomic volume of the implanted species. Thus detailed interatomic interactions are not included in the model, nor are the equilibrium shapes of the nanoclusters [22]. (Given the driven nature of the growth process, it is unlikely that nanoclusters assume their equilibrium shape during growth.)

Thermodynamic desorption from existing clusters is described by a local equilibrium approximation [3]. The desorption rate depends on the interface energy between the matrix and the implanted species, $\gamma$. Specifically, desorption from a cluster containing $s$-atoms is presumed to occur at a rate $1 / \tau_{s}$ :

$$
\frac{1}{\tau_{s}} \propto \sigma_{s} n_{\infty} \exp \frac{2 \gamma \Omega}{k T R_{s}}
$$

with $R_{s}$ the radius of a cluster containing $s$ atoms, $\sigma_{s}$ a capture length for clusters containing $s$ atoms, $n_{\infty}$ the solubility, and $k T$ Boltzmann's constant multiplied by temperature. Clusters are represented as spheres at all times. When atoms desorb/adsorb from/to existing clusters, the center of mass of the cluster and desorbed atom remains fixed.

Ion damage is modeled by extending the approach of Heinig and collaborators [9]. When a high energy ion impacts a solid, it leads to a collision cascade. The net effect of this cascade is to displace a large number of atoms in random directions. Heinig et al. suggest that this cascade be modeled by displacing the atoms within a cluster in random directions with distances $r$ governed by a Poisson distribution:

$$
p(r)=\frac{1}{8 \pi^{2} \lambda^{3}} \exp (-r / \lambda) .
$$

The value of $\lambda$ is then determined by fitting the displacement profiles for an ion-damaged embedded slab computed using TRIM [23]. Typical values of $\lambda$ range between 3 and $5 \AA$. 
An impinging ion hits existing nanoclusters with a probability proportional to the volume of the nanocluster, and the ion flux. When a cluster is struck, every atom in the cluster is displaced according to Eq. (2). The atoms are immediately checked to see if they "touch" other atoms. All "touching" atoms bind into or to clusters, and the simulation proceeds.

This coarse-grained description of the cascade is computationally efficient and it provides a good description of the results of a cascade. Specifically, molecular dynamics simulations of $\mathrm{Au}$ ions impacting free standing $\mathrm{Au}$ clusters yield a fragment size distribution that is characterized by a power law distribution of small fragments, $f_{s} \sim s^{-\alpha}$, with $f_{s}$ the number of fragments with $s$ atoms, and $\alpha=2.3$ [24]. Modeling the same process using KMC yields the same exponent. Further, the exponent appears to be independent of ion mass and energy.

A set of coupled rate equations (RE) are derived following references [2, 3, 21] while adding the effects of cluster fragmentation. Scaling all lengths by $L$ and all times by $T \equiv n_{\infty} / F$ the relevant equations become:

$$
\begin{aligned}
\frac{d\left\langle\tilde{n}_{1}\right\rangle}{d \tilde{t}} & =\tilde{F}-2 \tilde{\sigma}_{1}\left\langle\tilde{n}_{1}\right\rangle^{2}-\sum_{j>1} \tilde{\sigma}_{j}\left\langle\tilde{n}_{j}\right\rangle\left\langle\tilde{n}_{1}\right\rangle+2 \frac{\left\langle\tilde{n}_{2}\right\rangle}{\tilde{\tau}_{2}} \\
& +\sum_{j>2} \frac{\left\langle\tilde{n}_{j}\right\rangle}{\tilde{\tau}_{j}}+\tilde{F} \tilde{\Omega} \sum_{j>1}\left\langle\tilde{n}_{j}\right\rangle(j+1) K_{1}(\alpha, j),
\end{aligned}
$$

and

$$
\begin{aligned}
\frac{d\left\langle\tilde{n}_{s}\right\rangle}{d \tilde{t}} & =\tilde{\sigma}_{s-1}\left\langle\tilde{n}_{s-1}\right\rangle\left\langle\tilde{n}_{1}\right\rangle-\tilde{\sigma}_{s}\left\langle\tilde{n}_{s}\right\rangle\left\langle\tilde{n}_{1}\right\rangle-\frac{\left\langle\tilde{n}_{s}\right\rangle}{\tilde{\tau}_{s}} \\
& +\frac{\left\langle\tilde{n}_{s+1}\right\rangle}{\tilde{\tau}_{s+1}}-\tilde{F} \tilde{\Omega}\left\langle\tilde{n}_{s}\right\rangle(s+1) \\
& +\tilde{F} \tilde{\Omega} \sum_{j>s}\left\langle\tilde{n}_{j}\right\rangle(j+1) K_{s}(\alpha, j) .
\end{aligned}
$$

In Eqs. (3) and (4), $\left\langle\tilde{n}_{s}\right\rangle$ is the dimensionless average density of clusters containing $s$ atoms, $\tilde{\sigma}_{s}$ is the dimensionless capture length for an $s$-atom cluster to capture a diffusing atom. The dimensionless thermally activated desorption time for an atom to leave a cluster of $s$ atoms is $\tilde{\tau}_{s}$. The last terms of Eqs. (3) and (4) represent the fragmentation process: $\tilde{\Omega}$ is the dimensionless atomic volume of the implanted species and the term $\left\langle\tilde{n}_{j}\right\rangle \tilde{\Omega}(j+1)$ gives the probability of collision between an implanted atom and a $j$-cluster. $K_{s}(\alpha, j)$ is the number of $s$-clusters generated as a result of a framgented $j$-cluster:

$$
K_{s}(\alpha, j)=\left(\frac{j}{H_{j-1}^{1-\alpha}}\right) s^{-\alpha}, \text { with } \alpha=2.3,
$$


and $H_{j}^{r}$ the $j$ th harmonic number of order $r$.

For an initial calculation, we consider parameters near those appropriate for the implantation of Ge into amorphous silica. The initial volumetric implantation rate is set to be $F=10^{-6} \mathrm{sec}^{-1} \AA^{-3}$, roughly a factor of 7 larger than that employed by Sharp et al. [25], a choice made to enable reasonable computation times. TRIM calculations for $120 \mathrm{keV}$ Ge implanted into $\mathrm{SiO}_{2}$ give $\lambda=3.5 \AA$. The diffusion coefficient for Ge in $\mathrm{SiO}_{2}$ at room temperature is taken to be $D=6.5 \times 10^{-10} \mathrm{~cm}^{2} \mathrm{~s}^{-1}$ while the solid solubility is $n_{\infty}=10^{8}$ $\mathrm{cm}^{-3}$, both approximately equilibrium values [26]. Experimental studies stress relaxation [11] indicate that the $\mathrm{Ge} / \mathrm{SiO}_{2}$ interface energy is near $0.7-0.9 \mathrm{~J} \mathrm{~m}^{-2}$. However, choosing interface energies this large increases the critical cluster size for nucleation, and the associated simulations require substantial computational resources. Thus the Ge/silica interface energy is set to $0.2 \mathrm{~J} \mathrm{~m}^{-2}$. These parameters enable predictions to be obtained while expending a reasonable amount of computer time.

Typical results are shown in Fig. 1. Figure 1(a) plots both the average radius of the clusters, $\langle R\rangle$,

$$
\langle R\rangle=\frac{1}{N} \sum_{i=1}^{N} R_{i}
$$

with the index $i$ running over all clusters, $R_{i}$ the radius of cluster $i$, and $N$ the total number of clusters, as well as the cluster number density as a function of time. The cluster number density reveals four growth regimes. In the earliest stage (before 0.1 seconds), the number of clusters increases rapidly. Between 0.1 and 30 seconds, the number density of clusters rises slowly, while the average radius of the clusters rises rapidly. At very early times ion damage to existing clusters is rare relative to other processes, and the growth process is similar to that observed in 2-D nucleation and growth models. The onset of ion damage to clusters is marked by the peak in $\langle R\rangle$, and leads to a decrease in average cluster size. For times larger than 30 seconds, the clusters continue to be damaged, the average radius decreases, and the volume fills rapidly with clusters [Figs. 1(b) and 1(c)]. In the final stages of growth $\langle R\rangle$ approaches a steady-state value (not shown).

Figure 2 compares KMC and the rate equation results for the evolution of a cluster size distribution during implantation. The two computational approaches display qualitative agreement during the first two stages of growth. As indicated above, in the first stage of 
(a)

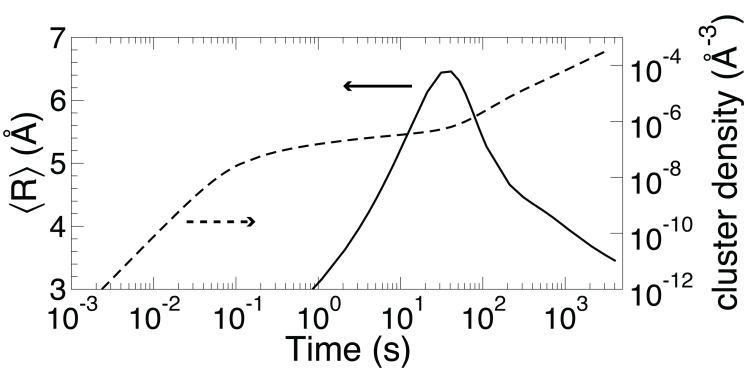

(b)

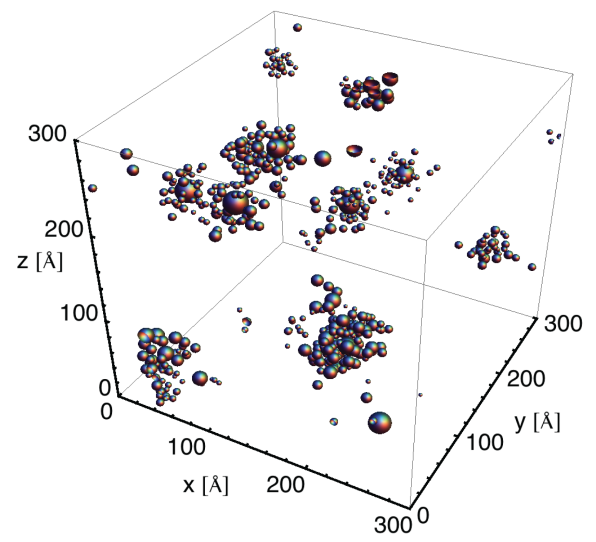

(c)

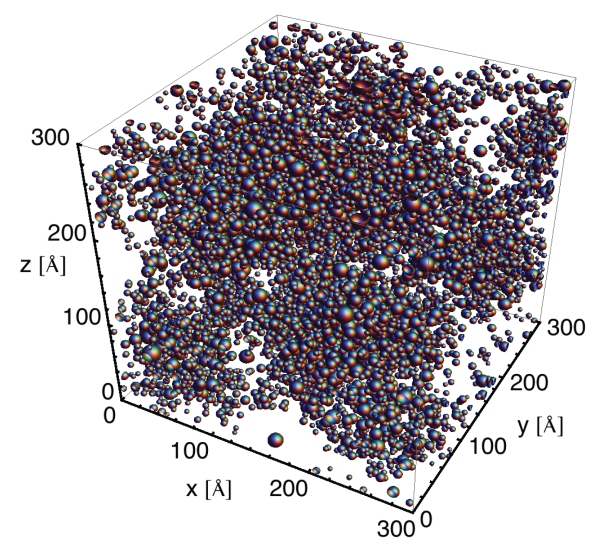

FIG. 1: (a) The average cluster radius (solid line, left axis) and the cluster number density (dashed line, right axis) as a function of time for typical growth conditions, (b) at 300 seconds and (c) at 3000 seconds.

growth the effect of damage cascade is negligible. The cluster size distribution attains a characteristic swept forward shape. As more mass is introduced into the system, fragmentation events occur more frequently and a peak representing the small fragments appears. This peak continues to grow and eventually dominates, rendering a unimodal distribution weighted at smaller sizes. 


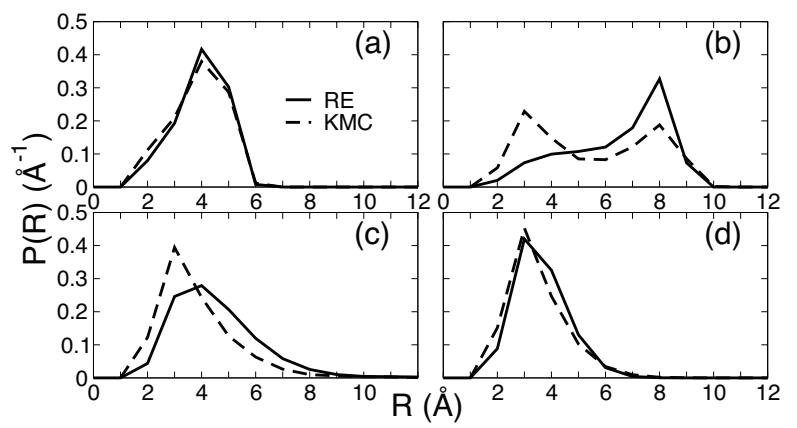

FIG. 2: KMC (dashed line) and RE (solid line) size distributions at (a) 3 seconds, (b) 30 seconds, (c) 300 seconds and (d) 3000 seconds for the same parameters as the plots shown in Fig. 1. The data from the rate equations has been binned using the same binwidth as used for KMC simulations in order to facilitate comparison.

It is observed empirically that the cluster size distribution reaches a steady-state. As this limit is approached, the shape of the cluster size distribution changes slowly and the steady-state shape serves as a good description of the shape of the cluster size distribution during the latter stages of implantation.

In $s$-space, the scaled steady-state cluster-size distribution, $g\left(\frac{s}{\bar{s}}\right)[21]$, can be found by solving:

$$
\begin{aligned}
& 0=\frac{d}{d t} g\left(\frac{s}{\bar{s}}\right)=\frac{d}{d t}\left(\bar{s} \frac{s\left\langle n_{s}\right\rangle}{F t}\right), \text { with } \\
& \bar{s}=\frac{1}{F t} \sum_{s=2}^{\infty} s^{2}\left\langle n_{s}\right\rangle
\end{aligned}
$$

in the limit that $\frac{d \bar{s}}{d t} \approx 0$. Solving these equations directly is efficient and accurate, and allows exploration of a broader range of parameters in comparison with integrating Eqs. (3) and (4).

Combining Eqs. (7) with Eqs. (3) and (4) and solving for large times yields the steadystate cluster size distribution. After conducting intermediate asymptotic analyses according to [27], one concludes that the steady-state solution depends on two parameters: the dimensionless atomic volume, $\tilde{\Omega}=\Omega / L^{3}$, and the interface energy, $\gamma$. In practice, however, we find that the steady-state shape of the distribution depends only on $\tilde{\Omega}$ and the average size depends on $\gamma$ (for a given temperature). This behavior is in marked contrast to the behavior observed during 2-D epitaxial growth in which the distribution shape is determined by the critical cluster size, a quantity that depends sensitively on $\gamma$. 
Experimentally measured as-implanted size distributions can be fitted to theory to obtain an estimate for the value of $L$. Figure 3 displays the fitted curves for three materials. Ge is best fit with a value of $L=1.38 \AA$, Co is described by $L=0.08 \AA$, and $\mathrm{Ag}$ is described by $L=2.66 \AA[30]$. For the case of Ge, assuming that coarsening is very slow (as is observed experimentally [10]), we can compare the observed value with the expected value. Using the accepted (normal) diffusion coefficient for Ge in silica one predicts $L=0.052 \AA$. The measured value for $L$ exceeds the initial prediction by a factor of $\approx 26.4$. The implication is that the ratio $D n_{\infty} / F$ exceeds the expected value by a factor of $\approx 700$. This increase is most likely due to ion damage within the matrix and is within the range expected for transient enhanced diffusion (TED) $[19,20]$. Hence, the values of $L$ needed to model experiments are reasonable. Further, measurements of the shape of the cluster size distribution can be used to determine the value of the transient enhanced diffusion coefficient during implantation.

It is unclear at this point how the value of $L$ varies with dose during implantation. The value of solid solubility in $\mathrm{SiO}_{2}$ is difficult to assess at temperatures relevant to IBS [26]. While little research has been done on TED in $\mathrm{SiO}_{2}$, studies of $\mathrm{B}$ implanted into Si suggest saturation of TED at doses greater than $\approx 3 \times 10^{14} \mathrm{~cm}^{-2}$ [28], roughly $1 \%$ of typical IBS doses. We expect, therefore, that for the systems studied here, $L$ changes rapidly during the initial stages of growth, but quickly stabilizes to the value deduced from the steady state distributions. Further, ion damage serves to minimize the influence of the initial implantation performed during the stages with rapidly varying $L$.

The theory also suggests how one might narrow IBS cluster-size distributions. Figure 3(b) plots the computed full-width at half-maximum, scaled by the average cluster radius, $\Delta R /\langle R\rangle$, as a function of $L$ for Ge. As $L$ decreases, so does the relative width. Near $L \approx 1$ $\AA$ the curve shows a sharp downward trend. Experimentally, $L$ can be decreased by increasing the flux of ions and/or decreasing the transient enhanced diffusion, perhaps by cooling $[19,29]$.

In conclusion, a theory for the shape of the cluster-size distributions arising during IBS is introduced and compared with the results of kinetic Monte Carlo simulations of the growth. The theory predicts that the nanocluster size distribution asymptotes to a steady state. The steady-state shape of the nanocluster size distribution depends only on $\tilde{\Omega}$, whereas 

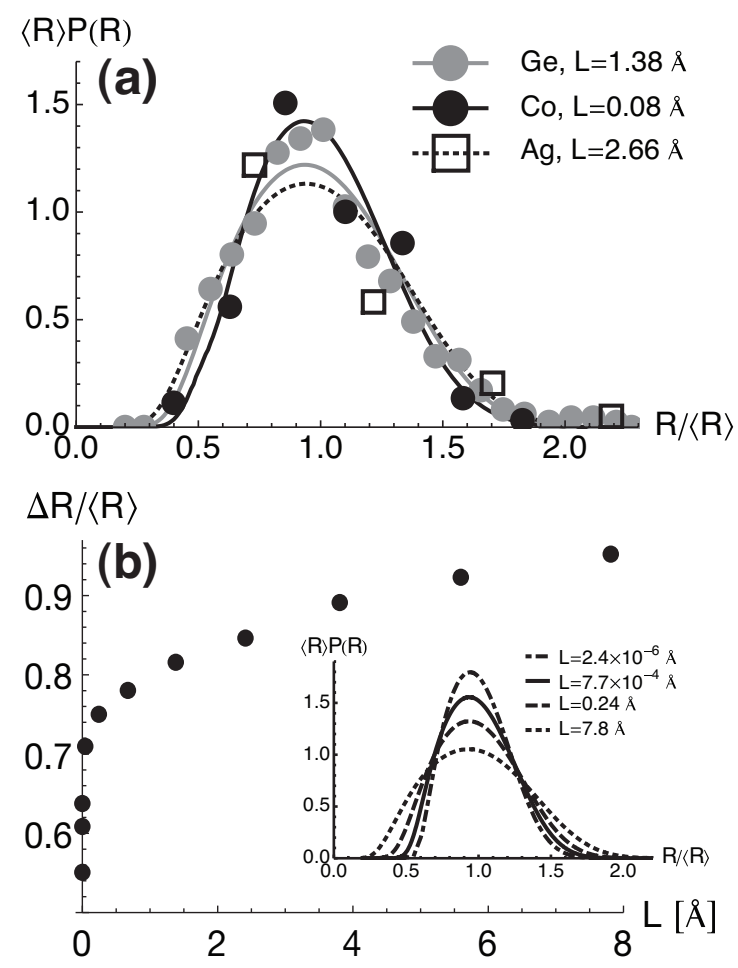

FIG. 3: (a) Steady-state shape of the nanocrystal size distribution function obtained by fitting theory to experimental room-temperature as-implanted size distributions for $\mathrm{Ge}, \mathrm{Ag}$ [13], and $\mathrm{Co}$ [12]. The fits provide an excellent description of the available data. (b) Full-width at half maximum plotted as a function of $L$ predicted by the model. The inset shows the steady-state cluster size distributions expected for different $L$.

the average size cluster is determined by the interface energy. The theory can be used to measure transient enhanced diffusion coefficients during implantation, and to develop processing routes to narrow cluster-size distributions.

This research is supported by the Directorate, Office of Science, Office of Basic Energy Sciences, Division of Materials Sciences and Engineering, of the U.S. Department of Energy under Contract No. DE-AC02-05CH11231.

[1] L. Ratke and P. W. Voorhees, Growth and Coarsening (Springer-Verlag, New York, 2002), 1st ed.

[2] G. S. Bales and D. C. Chrzan, Phys. Rev. B 50, 6057 (1994). 
[3] G. S. Bales and A. Zangwill, Phys. Rev. B 55, R1973 (1997).

[4] J. A. Stroscio and D. T. Pierce, Phys. Rev. B 49, 8522 (1994).

[5] J. G. Amar and F. Family, Physical Review Letters 74, 2066 (1995).

[6] C. Ratsch, A. Zangwill, P. Smilauer, and D. D. VVedensky, Phys. Rev. Lett. 72, 3194 (1994).

[7] Y. W. Mo, J. Kleiner, M. B. Webb, and M. G. Lagally, Physical Review Letters 66, 1998 (1991).

[8] A. Pimpinelli, J. Villain, and D. E. Wolf, Physical Review Letters 69, 985 (1992).

[9] K. H. Heinig, T. Müller, B. Schmidt, M. Strobel, and W. Möller, Appl. Phys. A 77, 17 (2003).

[10] Q. Xu, I. D. Sharp, C. W. Yuan, D. O. Yi, A. M. G. C. Y. Liao, A. M. Minor, J. W. Beeman, M. C. Ridgway, J. W. A. III, D. C. Chrzan, et al., Phys. Rev. Lett. 97, 155701 (2006).

[11] I. D. Sharp, D. O. Yi, Q. Xu, C. Y. Liao, J. W. Beeman, Z. Liliental-Weber, K. M. Yu, D. Zhakarov, J. W. A. III, D. C. Chrzan, et al., Applied Physics Letters 86, 063107 (2005).

[12] E. Cattaruzza, F. Gonella, G. Mattei, P. Mazzoldi, D. Gatteschi, C. Sangregorio, M. Falconieri, G. Salvetti, and G. Battaglin, Appl. Phys. Lett. 73, 1176 (1998).

[13] C. Z. Jiang and X. J. Fan, Surface and Coatings Technology 131, 330 (2000).

[14] G. D. Marchi, G. Mattei, P. Mazzoldi, and C. Sada, J. Appl. Phys 92, 4249 (2002).

[15] R. Giulian, P. Kluth, L. L. Araujo, D. J. Llewellyn, and M. C. Ridgway, Appl. Phys. Lett. 91, 093115 (2007).

[16] V. Ramaswamy, T. E. Haynes, C. W. White, W. J. MoberlyChan, S. Roorda, and M. J. Aziz, Nanoletters 5, 373 (2005).

[17] G. Dearnaley, Nature 256, 701 (1975).

[18] T. Shinada, S. Okamoto, T. Kobayashi, and I. Ohdomari, Nature 437, 1128 (2005).

[19] N. E. B. Cowern and C. S. Rafferty, MRS Bulletin 25, 39 (2000).

[20] P. A. Stolk, H.-J. Gossmann, D. J. Eaglesham, D. C. Jacobson, C. S. Rafferty, G. H. Gilmer, M. Jaraiz, J. M. Poate, H. S. Luftman, and T. E. Haynes, J. Appl. Phys. 81, 6031 (1997).

[21] D. O. Yi, M. H. Jhon, I. D. Sharp, Q. Xu, C. W. Yuan, C. Y. Liao, J. W. A. III, E. E. Haller, and D. C. Chrzan, Phys. Rev. B 78, 245415 (2008).

[22] F. Baletto and R. Ferrando, Rev. Mod. Phys 77, 371 (2005).

[23] J. F. Ziegler, J. P. Biersack, and U. Littmark, The Stopping and Range of Ions in Solids (Pergamon Press, New York, 1985).

[24] R. Kissel and H. M. Urbassek, Nucl. Inst. Meth. Phys. Res. B 180, 293 (2001). 
[25] I. D. Sharp, Q. Xu, C. Y. Liao, D. O. Yi, J. W. Beeman, Z. Lilienthal-Weber, K. M. Yu, D. N. Zakharov, J. W. A. I. an d D. C. Chrzan, and E. E. Haller, J. Appl. Phys. 97, 124316 (2005).

[26] J. D. McBrayer, R. M. Swanson, and T. W. Sigmon, J. Electrochem. Soc. 133, 1242 (1986).

[27] G. I. Barenblatt, Scaling (Cambridge University Press, 2003), 1st ed.

[28] N. E. B. Cowern, K. T. F. Janssen, and H. F. F. Jos, J. Appl. Phys. 68, 6191 (1990).

[29] L. Pelaz, G. H. Gilmer, V. C. Venezia, H.-J. Gossman, M. Jaraiz, and J. Barbolla, Appl. Phys. Lett. 74, 2017 (1999).

[30] Due to the small number of points describing this distribution, the uncertainty in the value of $L$ for $\mathrm{Ag}$ is substantial. 